\title{
Zero Flux Eigenvalues (ZFE's) of Tight Aspect Ratio Tokamaks
}

\author{
Sherif Mohamed Khalil1,2, Noura Ali Alomayrah1 \\ ${ }^{1}$ Faculty of Science, Physics Department, Princess Nora Bint Abdurrahman University, Riyadh, Kingdom of Saudi \\ Arabia \\ ${ }^{2}$ On Leave from Plasma Physics Department, Nuclear Research Center, Atomic Energy Authority, Cairo, Egypt \\ Email: smkhalil@pnu.edu.sa
}

Received 29 August 2015; accepted 3 January 2016; published 6 January 2016

Copyright (C) 2016 by authors and Scientific Research Publishing Inc.

This work is licensed under the Creative Commons Attribution International License (CC BY). http://creativecommons.org/licenses/by/4.0/

(c) (i) Open Access

\section{Abstract}

Compact toroidal magnetized plasmas are an important part of the world's magnetic fusion and plasma science efforts. These devices can play an integral role in the development of magnetic fusion as a viable commercial energy source, and in our understanding of plasma instabilities, particle and energy transport, and magnetic field transport. In this paper, we are developing a numerical program to study the magnetic dynamo or relaxation of CT's characterized by arbitrary tight aspect ratio (major to minor radii of tokamak) and arbitrary cross-sections (Multi-pinch and D-Shaped). The lowest ZFE's has been calculated through the Taylor's relaxed state (force-free) toroidal plasmas equation. For ZFE's, we use the toroidal flux vanishing boundary condition along the whole boundary of tokamaks. Several runs of the program for various wave numbers showed that ZFE was very insensitive to the choice of wave numbers. Besides, the CT's poloidal magnetic field topologies are well represented. It was very interesting to check our methods for the cases when aspect ratio tends to unity (zero tokamak whole). A good fulfillment of the boundary condition is achieved.

\section{Keywords}

Relaxed Plasma State, Compact Tokamaks

\section{Introduction}

Compact toroidal magnetized plasmas became recently an important part of the world's magnetic fusion and plasma science efforts. These devices can play an integral role in the development of magnetic fusion as a viable commercial energy source, and in our understanding of plasma instabilities, particle and energy transport, and 
magnetic field transport.

Compact toroid (CT) configuration is of simpler construction than the conventional tokamak and has important advantages due to the novel physics properties of its low aspect ratio. It could afford smaller, less-expensive fusion power plants, so it is becoming increasingly popular. There are several worldwide types of compact toroids as spheromaks: field reversed configuration (FRC), and other compact toroidal devices which had achieved remarkable successful results via fusion plasma parameters.

These devices can play an integral role in the development of magnetic fusion as a viable commercial energy source, and in our understanding of plasma instabilities, particle and energy transport, and magnetic field transport, but still there is a need to develop an experimental programs to study some of these phenomena with emphasis on transport, the relaxation state, non-classical ion acceleration and magnetic reconnection [1] [2].

For reversed field pinch and spheromak configuration, magnetic helicity has been believed to play an important role as a global invariant in the process of self-organization and relaxation for magnetized plasma in CT. Accordingly, we have to go back to J. B. Taylor model [3] [4]

$$
\boldsymbol{J}=\nabla \times \boldsymbol{B}=\mu \boldsymbol{B},
$$

to study and investigate the relaxed state (force-free) for compact to kamaks. The theory has been successfully applied to many fusion related plasmas, especially CT's.

The steady state equilibrium of CT is characterized by $\nabla P=\boldsymbol{J} \times \boldsymbol{B}$, which represents the general form of Grad-Shafranov equation. Simply, an expression of the static pressure balances between gradient in kinetic pressure and magnetic forces.

Compact tori are typically characterized by low $\beta$, and accordingly simplest equilibrium model is given at vanishing the plasma gradient ( $\nabla P=0$ ), i.e., the current density $\boldsymbol{J}$ is completely parallel to the magnetic field $\boldsymbol{B}$, and also vanishing of magnetic stress $(\boldsymbol{J} \times \boldsymbol{B}=0)$. Accordingly the equilibrium equation reduces to Taylor's relaxed state equation:

$$
\nabla \times \boldsymbol{B}=\mu \boldsymbol{B}, \mu=\text { const. }
$$

Equation (1) describes the relaxed toroidal plasmas to a force-free configuration of minimum energy. It was a representation of the convential Beltrami equilibrium, which also restricted to only force-free equilibrium.

If $\mu$ is spatially uniform, then the curl of Equation (1) gives a vector Helmholtz equation, $\nabla^{2} \boldsymbol{B}+\mu^{2} \boldsymbol{B}=0$ [5]. The divergence of Equation (1) gives $\boldsymbol{B} \cdot \nabla \mu=0$ which means that $\mu$ is constant on a field line. In relaxed states $\mu$ cannot exceed the smallest eigenvalue $\mu_{\min }$ and for a toroidal discharge, there is a maximum toroidal current $I_{\max }$ which is connected to $\mu . \mu_{\min }$ satisfies a zero flux boundary condition equation:

$$
\iint B_{\varphi} \mathrm{d} r \cdot \mathrm{d} z=0
$$

which defines current limitation in tooidal systems.

Solutions of (1) are perceived to describe the gross features of FRC, spheromak configurations, current limitation in toroidal plasmas and others. Two parameters are determining the relaxed state for toroidal system with a perfectly conducting boundary. Firstly, is the magnetic helicity $\kappa\left(\kappa=\int \boldsymbol{A} \cdot \boldsymbol{B} \mathrm{d} \tau\right)$ and secondly is the toroidal flux $\psi . \mu$ is an very important inverse length parameter. Eigenvalue $\mu$ and the normalized field profile are determined by the dimensionless ratio $\kappa / \psi^{2}[6]$.

To select the correct minimum energy solution of Equations (1) and (2), one can consider the eigenvalues of this system [7]. Solutions of Equations (1) and (2) seem to be difficult in toroidal coordinates. Therefore, the force-free magnetic field boundary value problem has been solved in the toroidal coordinates using approximate analytical methods, considering a large, but finite, aspect ratio [8] [9]. Toroidal force-free equilibrium has also been considered precisely for tori of rectangular cross section and finite aspect ratio, and for more general boundaries in the infinite aspect ratio limit [7] [9].

The aim of present work is to reply to the questions which may be arise about the applicability of collocation methods [10]-[14] to investigate and study the relaxed plasma state for tight aspect ratio tokamaks with multipinch and D-shaped boundaries.

We calculated the lowest eigenvalues $\mu_{\min }$, which associated with relaxed force-free of the system. Furthermore, we proved the fulfillment of the boundary condition (2) along the whole boundary for different sharp edged cross-section. Dependence of $\mu$ on the aspect ratio is also studied. Besides, the magnetic field topologies inside the CT are represented. 


\section{Relaxed States Analytical Solutions for Axisymmetric CT}

In order to determine the minimum eigenvalues, we apply to equations (1) and (2) a method of solving equations of this type - collocation method [10] [15] [16] — for a torus of arbitrary cross-sections and tight aspect ratios. In toroidal container and for finite aspect ratio the periodicity condition is expressed as $\boldsymbol{B}(r, \varphi, z)=\boldsymbol{B}(r, \varphi+2 \pi, z)$. Let us assume also, a variables separation as $F(r, \varphi, z)=f(r) \exp i(m \varphi+k z)$.

According to Taylor [1] [2] [6], $\mu_{\min }$ will satisfy different sorts of boundary conditions.

(i) The boundary condition (which is applied to all cases): $|(\boldsymbol{B} \cdot \boldsymbol{n})|_{\text {wall }}=0$, it follows that $\left|r B_{\varphi}\right|_{\text {Wall }}$ is constant if $\frac{\partial}{\partial \varphi}=0$ (axi-symmetric tokamak).

(ii) The zero field condition: $\left|B_{\varphi}\right|_{\text {Wall }}=0$ [10] [16].

(iii) The zero flux condition: $\iint B_{\varphi} \mathrm{d} r \mathrm{~d} z=0$ [17], which defines current limitation in tokamaks, and vanishing of azimuthal magnetic field.

For a non-axisymmetric mode the boundary condition (i) alone determine a zero-flux eigenvalue, the condition $|(\boldsymbol{B} \cdot \boldsymbol{n})|_{\text {wall }}=0$ is automatically ensure that $\iint B_{\varphi} \mathrm{d} r \mathrm{~d} z=0$.

For axisymmetric mode, the boundary condition (i) alone is not sufficient to determine an eigenvalue. The zero flux condition (iii) must be explicitly imposed to determine axisymmetric eigen-modes. The vanishing field condition (ii) is really an independent problem. It determines its own set of eigenvalues (the zero-field eigenvalues). The connection with the zero-flux problem arises because some (but not all) zero0field eigenvalues, defined by (ii), exactly coincide with some (but not all) zero-flux eigenvalues defined by (iii).

The meridional cross-section in the $r$, $z$ plane of the toroidal metallic vessel wall shall be described by an arbitrary curve $z=z(r)$ along which the boundary condition (2) has to be satisfied. Later, the curve $z=z(r)$ will be represented by a set of points (collocation points) $r, z$ which lay on the cross-section boundary.

From (1), the following expressions linking the magnetic field components are obtained:

$$
\begin{aligned}
& i \frac{m}{r} B_{z}-i k B_{\varphi}=\mu B_{r}, \\
& i k B_{r}-\frac{\partial B_{z}}{\partial r}=\mu B_{\varphi}, \\
& \frac{1}{r} \frac{\partial\left(r B_{\varphi}\right)}{\partial r}-i \frac{m}{r} B_{r}=\mu B_{z}
\end{aligned}
$$

As the toroidally non-axisymmetric states are not so evident in the experiments, we concentrate mainly on axisymmetric or $\varphi$ independent states.

Solution of Equations (3) gives the magnetic field components for axisymmetric toroidal plasmas as:

$$
\begin{gathered}
B_{r}(r, z)=\sum_{l=1}^{N} \frac{k_{l}}{\chi_{l}} \sin \left(k_{l} z\right)\left[a_{l} J_{1}\left(\chi_{l} r\right)+b_{l} Y_{1}\left(\chi_{l} r\right)\right] \\
B_{z}(r, z)=\sum_{l=1}^{N} \cos \left(k_{l} z\right)\left[a_{l} J_{0}\left(\chi_{l} r\right)+b_{l} Y_{0}\left(\chi_{l} r\right)\right] \\
B_{\varphi}(r, z)=\sum_{l=1}^{N} \frac{\mu}{\chi_{l}} \cos \left(k_{l} z\right)\left[a_{l} J_{1}\left(\chi_{l} r\right)+b_{l} Y_{1}\left(\chi_{l} r\right)\right]
\end{gathered}
$$

where, $J_{0}, J_{1}$ are Bessel functions; $Y_{0}, Y_{1}$ are Neumann functions; $a_{l}, b_{l}$ are constant coefficients, and $\chi_{l}=\sqrt{\mu^{2}-k_{l}^{2}}$.

It should be mentioned here that, for the case of a straight cylinder (or for infinite aspect ratio of toroidal plasma), the coefficient $b_{l}$ goes to zero, since the Neumann functions are singular for $r=0$. On the other hand, for finite aspect ratio or CT, as in our case, one has to keep these functions.

Inserting (5) into (2), the zero flux boundary condition for an axisymmetric container of finite aspect ratio and arbitrary cross-section reads [17]: 


$$
\sum_{l=1}^{N} \frac{\mu}{k_{l} \chi_{l}^{2}} \sin \left(k_{l} z\right)\left[a_{l} J_{0}\left(\chi_{l} r\right)+b_{l} Y_{0}\left(\chi_{l} r\right)\right]=C
$$

\section{Numerical Solution}

Plasma is an inhomogeneous anisotropic nonlinear medium and modeling of plasma devices and the solutions of the associated mathematical problems are not a simple task. Many plasma machines are of toroidal geometry, hence their associated physical processes are described by partial differential equations with space dependent coefficients. Often these equations can not be separated in ordinary differential equations and one has to take refuge with numerical methods. The large computer CODES have been developed and worked quite well. However these codes showed disadvantages via small and compact tokamaks (characterized by great curvature or small aspect ratio). Should we arrive that a partial differential equations is not separable in a special coordinate system, then they will be solved in a coordinate system in which it is separable. This offers the great advantages that the boundary curves can be described by another coordinate systems or by a set of collocation points and that this description is absolutely independent of the coordinate system in which the partial differential equations is separable.

The collocation method [10] [11] may be applied to compact tori of Cassini (multi-pinch) and D-Shaped meridional cross-sections and arbitrary tight aspect ratio to calculate the lowest eigenvalue $\mu_{\min }$ and to investigate the CT's poloidal magnetic field topologies are well represented.

\subsection{Cassini Cross-Section (Multi-Pinch Compact Device)}

For tokamak with multi-pinch cross section, the meridional curve is given by:

$$
Z_{i}=\sqrt{\sqrt{a^{4}-4 b^{2}\left(r_{i}-R\right)^{2}}+b^{2}-\left(r_{i}-R\right)^{2}}
$$

Thus, $Z_{i}$ in (7) may be expressed by (8). Numerically, for mode $\mathrm{N}=4$, and arbitrary aspect ratio $\alpha=1-20$, the obtained results for zero flux eigenvalues $\mu$ against the aspect ratio $\alpha$ are given by Table 1 .

This shows that, by increasing aspect ratio $\alpha$, the zero flux eigenvalue is slightly increases.

All our results have been calculated for a multi-pinch cross section given by the curve (8). This curve, along which the boundary condition is fulfilled, must also be described by the zero flux boundary condition (7). For C $=0$, this gives the poloidal magnetic field lines along the container. Besides, for $C$ has different values, this gives the poloidal magnetic flux lines inside the container. The toroidal shift (Shafranov's shift) is clearly seen and depends on the aspect ratio, as per Figure 1.

The collocation points $z_{i}, r_{i}$ are marked by small black circles, and symmetrically distributed over the whole upper half of the cross-section.

Besides, Figure 2, shows the magnetic field components $B_{r}(r, z), B_{z}(r, z)$ and $B_{\varphi}(r, z)$ for CT with large and small elongation multi-pinch cross section.

\subsection{D-Shape Cross-Section}

Let us consider now a tokamak with D-Shaped cross section with dimensions: $R=1.2, a=0.6, \alpha=R / a=2$, and collocation points given in Table 2.

Table 1. Zero flux eigenvalues $\mu$ against the aspect ratio $\alpha(\mu$ vs $\alpha)$.

\begin{tabular}{ccccccc}
\hline$\alpha=\mathrm{R} / \mathrm{a}$ & 1 & 1.2 & 1.5 & 2 & 5 & 20 \\
\hline$\mu$ & 2.0338 & 2.03967 & 2.04409 & 2.04733 & 2.05064 & 2.05119 \\
\hline
\end{tabular}

Table 2. D-Shaped cross section with dimensions.

\begin{tabular}{cccccccccc}
\hline$r_{i}$ & 0.60 & 0.60 & 0.60 & 0.92 & 1.20 & 1.48 & 1.76 \\
\hline$z_{i}$ & 0.01 & 0.44 & 0.99 & 0.99 & 0.91 & 0.73 & 0.28 \\
\hline
\end{tabular}



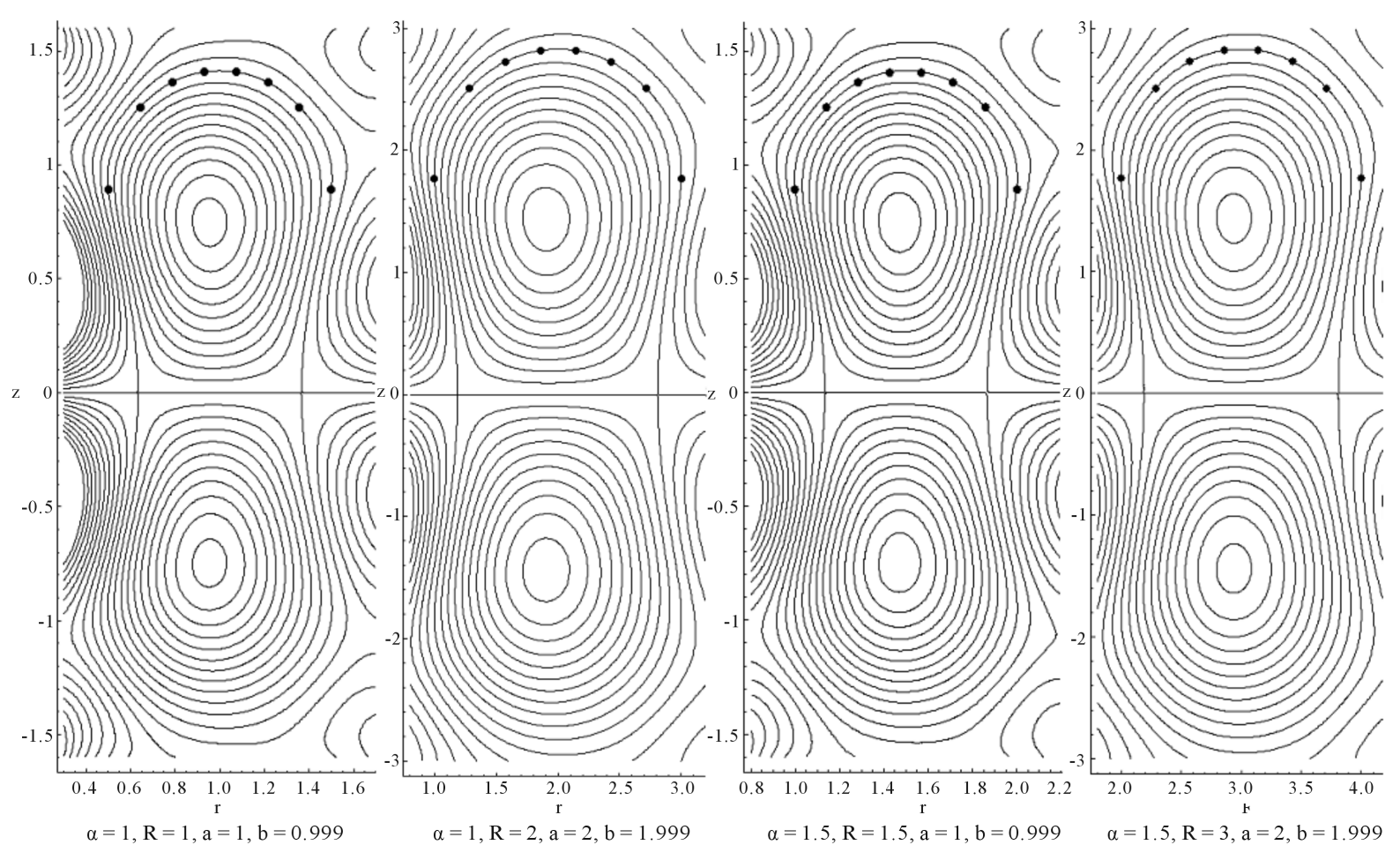

Figure 1. Poloidal magnetic flux in multi-pinch cross-sections for arbitrary aspect ratio.

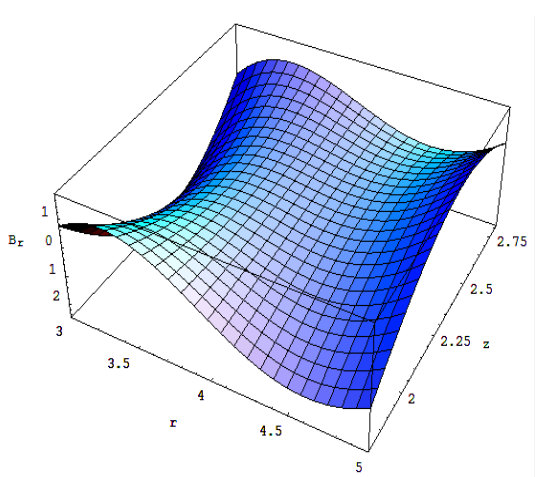

$B_{r}(r, z)$

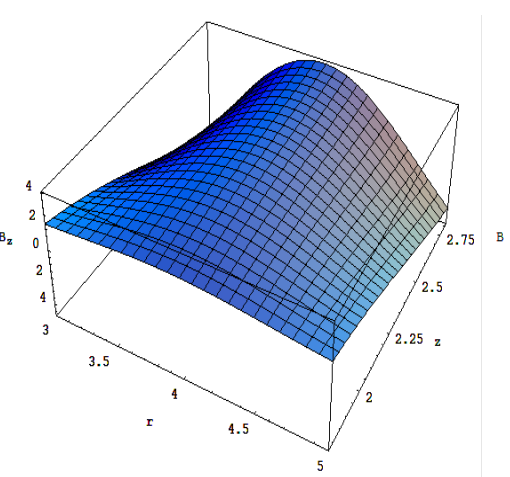

$B_{z}(r, z)$

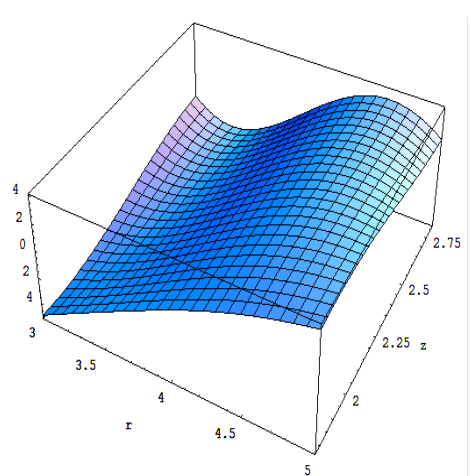

$B_{\varphi}(r, z)$

Magnetic field components for large elongation multi-pinch cross section with $\alpha=2, R=4, a=2, b=1.999$

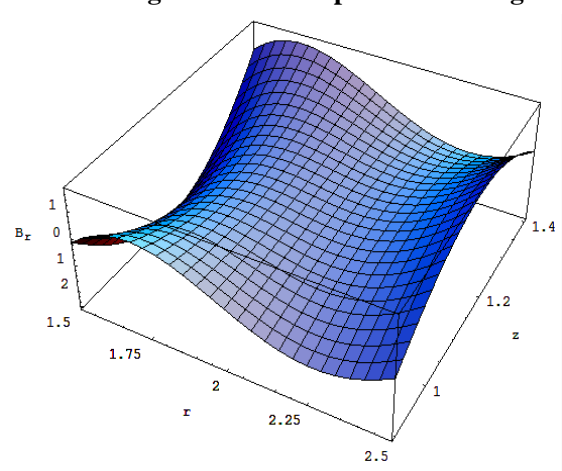

$B_{r}(r, z)$

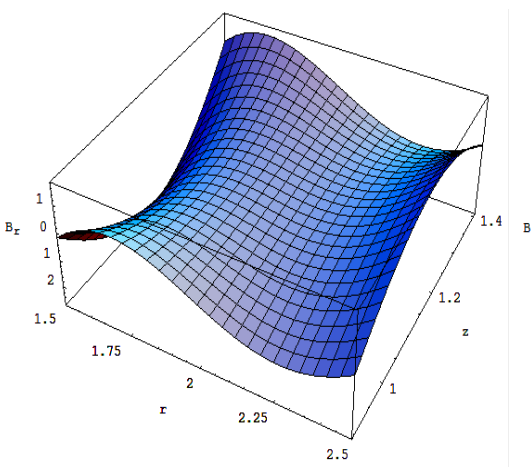

$B_{z}(r, z)$

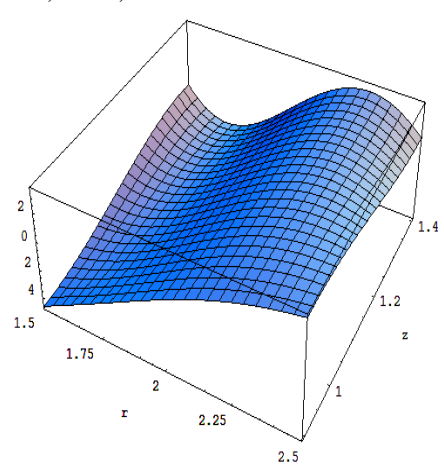

$B_{\varphi}(r, z)$

Magnetic field components for smallzongation multi-pinch cross section with $\alpha=2, R=2, a=1, b=0.999$

Figure 2. The magnetic field components $B_{r}(r, z), B_{z}(r, z)$ and $B_{\varphi}(r, z)$ for CT with large and small elongation multi-pinch cross section. 
For different arbitrary aspect ratio $\alpha$, mode $N=4$, and $k$-values ( $k=4.3999,3.42212,2.44434,1.46657$ ), we obtained the zero flux eigenvalues as per Table 3.

Applying the zero flux condition (7), for $C=0$, and different values of $C$, we get the following poloidal magnetic field picture inside the $\mathrm{D}$-shaped cross-sections for arbitrary aspect ratio (Figure 3 ).

Besides, Figure 4, shows the magnetic field components $B_{r}(r, z), B_{z}(r, z)$ and $B_{\varphi}(r, z)$ for CT's with aspect ratios $(\alpha=3 ; \alpha=1.1)$.

\section{Discussions and Conclusions}

The paper presents a method for determining the ZFE's of relaxed plasma states in compact tori of Multi-pinch and D-Shaped cross-sections.

If plasma is approximately force-free and approximately in equilibrium, then its magnetic field is determined by the simple force-free equation $\nabla \times \boldsymbol{B}=\mu \boldsymbol{B}$. The behaviour of the solutions to this equation is determined by boundary conditions and by the internal $\mu$ profile. CT's like Spheromak is distinguished by its differing boundary conditions: spheromaks have azimuthally symmetry whereas prominences have mirror symmetry about a plane.

As shown in Table 1 and Table 2, for both Multi-Pinch and D-shaped tokamaks, the aspect ratio $\alpha$ is found to play a crucial role in finding the ZFE's, hence it strongly affects the physical picture of toroidal plasmas, specially when speaking about the current limitation in tokamaks. The lowest ZFE's is obtained for $\alpha \rightarrow 1$.

It is also shown that the numerical method used here, works quite well for (CT) with tight aspect ratio (Figure 1 and Figure 3). It gives, with high accuracy, the ZFE's of the relaxed force-free equation. A good fulfilment of the boundary condition (7), which described the relaxed state along the whole boundary for different aspect ratios, is achieved even for aspect ratio approaches unity $(\alpha \rightarrow 1)$.

The toroidal shift (Shafranov's shift) is clearly seen and depends on the aspect ratio, as per Figure 1.

Recently, the problem studied in this paper, Taylor/Beltrami relaxation, is still finding a great interest [16][21].

Table 3. The zero flux eigenvalues ( $\mu$ vs $\alpha$ ).

\begin{tabular}{ccccccc}
\hline$\alpha=R / a$ & 1.05 & 1.1 & 2 & 5 & 10 & 20 \\
\hline$\mu$ & 4.40478 & 4.43098 & 4.55571 & 4.57714 & 4.57964 \\
\hline
\end{tabular}
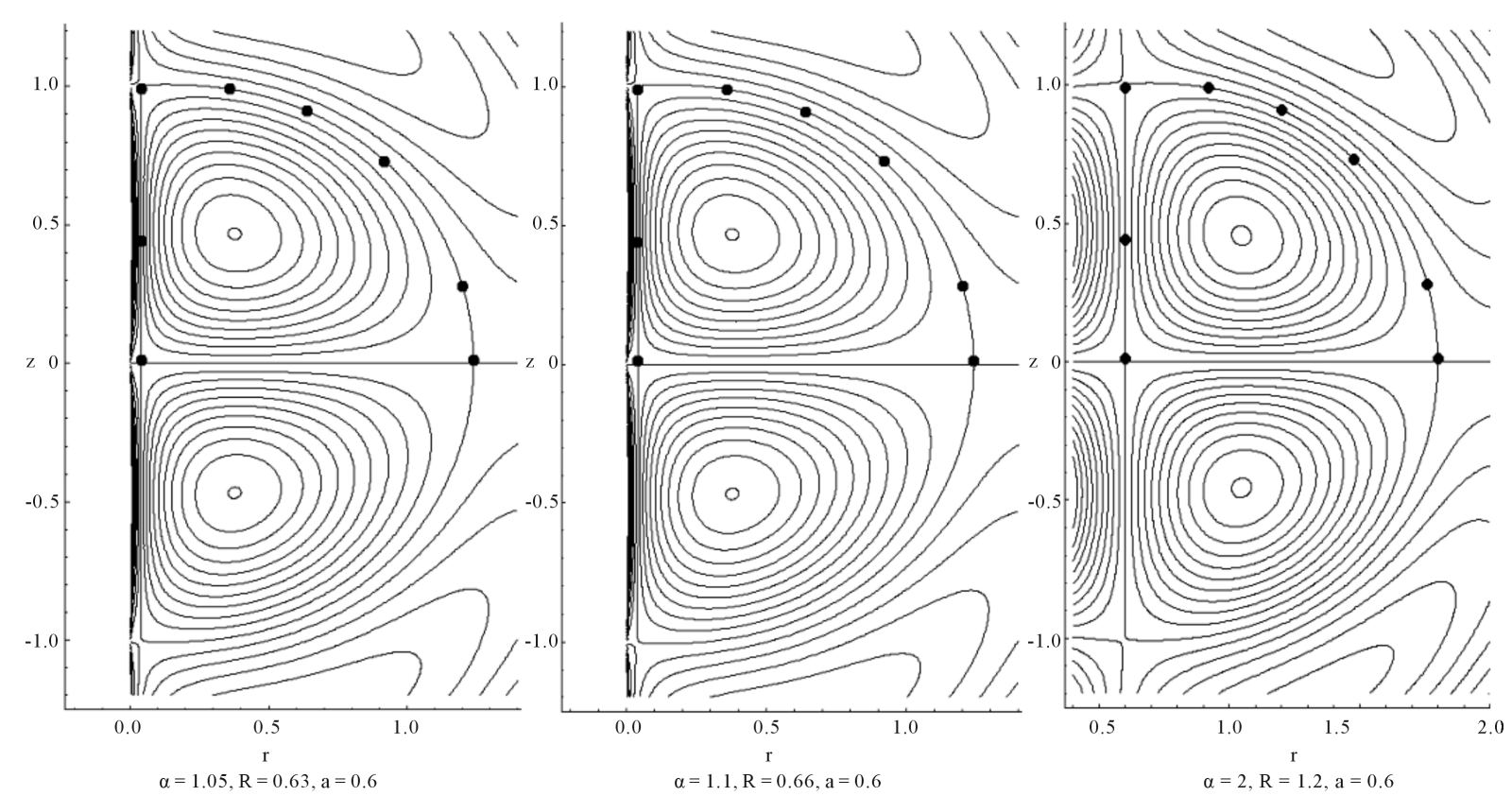

Figure 3. The D-shaped cross-sections for arbitrary aspect ratio. 

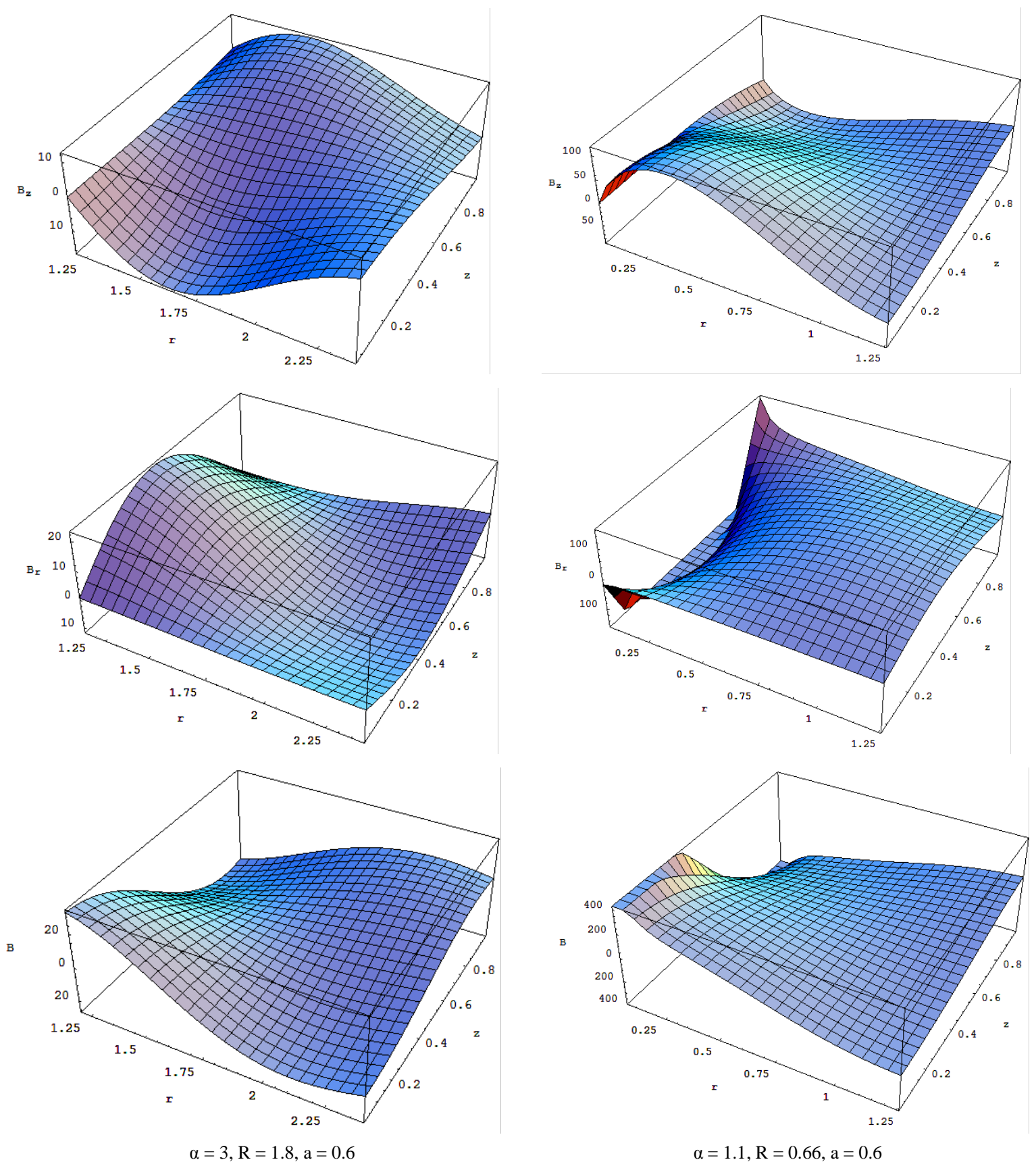

Figure 4. The magnetic field components $B_{r}(r, z), B_{z}(r, z)$ and $B_{\varphi}(r, z)$ for CT's with aspect ratios $(\alpha=3 ; \alpha=1.1)$.

Low aspect ratio plasmas in devices such as the mega ampere spherical tokamak (MAST) are characterized by strong toroidicity, strong shaping and self fields, low magnetic field, high beta, large plasma flow and high intrinsic $E \times B$ flow shear. These characteristics have important effects on plasma behaviour, provide a stringent test of theories and scaling laws and offer new insight into underlying physical processes, often through the amplification of effects present in conventional tokamaks (e.g. impact of fuelling source and magnetic geometry on $\mathrm{H}$-mode access) [22]. Compared to higher aspect ratio $\alpha$, low $\alpha$ leads to modest toroidal field and high plasma current in a small size which reduces weight and capital cost for the fusion power core.

Strong plasma shaping can lead to high steady state confinement and stable beta which ensure ignited burn capability in this plasma. Normal conducting toroidal field coils that dissipate power become acceptable under these conditions. High self-driven current reduces external current drive and recirculating power. 
An interesting point is arised to consider in future the coupling magnetic and fluid aspects of plasma (i.e., for $\boldsymbol{J} \times \boldsymbol{B} \cong \nabla P \neq 0$ ) .

\section{References}

[1] Joint Meeting of the $3^{\text {rd }}$ IAEA Technical Meeting on Spherical Tori and the $11^{\text {th }}$ International Workshop on Spherical Torus, 3-6 October 2005. http://www.apmath.spbu.ru/iaea/

[2] NI Lecture: Fusion Energy: Can Smaller Be Better? Colin Windsor, Tokamak Energy, 2015. https://goo.gl/ZHZT6

[3] Taylor, J.B. (1974) Relaxation of Toroidal Plasma and Generation of Reverse Magnetic Fields. Physical Review Letters, 33, 1139. http://dx.doi.org/10.1103/PhysRevLett.33.1139

[4] Taylor, J.B. (1986) Relaxation and Magnetic Reconnection in Plasmas. Reviews of Modern Physics, 58, 741. http://dx.doi.org/10.1103/RevModPhys.58.741

[5] Bellan, P.M., Yee, J. and Hansen, J.F. (2001) Spheromaks, Solar Prominences, and Alfven Instability of Current Sheets. Earth Planets Space, 53, 495. http://dx.doi.org/10.1186/BF03353261

[6] Taylor, J.B. and Turner, M.F. (1989) Plasma Current Drive by Helicity Injection in Relaxed States. Nuclear Fusion, 29, 1166. http://dx.doi.org/10.1088/0029-5515/29/7/009

[7] Gimblett, C.G., Hall, P.J., Taylor, J.B. and Turner, M.F. (1987) Eigenvalues Associated with Relaxed States of Toroidal Plasmas. Physics of Fluids, 30, 3186. http://dx.doi.org/10.1063/1.866494

[8] Buck, G.J. (1965) Force-Free Magnetic-Field Solution in Toroidal Coordinates. Journal of Applied Physics, $36,2231$. http://dx.doi.org/10.1063/1.1714456

[9] Turner, M.F. (1984) Analytic Solutions of $\nabla \times B=\lambda B$ Having Separatrices for Geometries with One Ignorable Coordinate. Physics of Fluids, 27, 1677. http://dx.doi.org/10.1063/1.864822

[10] Cap, F. and Khalil, Sh.M. (1989) Eigenvalues of Toroidal Plasmas of Arbitrary Aspect Ratio and Arbitrary Cross Section. Nuclear Fusion, 29, 1166. http://dx.doi.org/10.1088/0029-5515/29/7/009

[11] Cap, F. (1986) Electromagnetic Eigenfrequency of Anisotropic Inhomogeneous Axisymmetric Toroidal Plasmas of Arbitrary Meridional Cross Section. Computer Physics Communications, 40, 99-103. http://dx.doi.org/10.1016/0010-4655(86)90151-7

[12] Cap, F. (1986) Boundary Value Problems of Unseparable Linear Partial Differential Equations. Journal of Applied Mathematics and Mechanics (ZAMM), 66, 248-250. http://dx.doi.org/10.1002/zamm.19860660612

[13] Cap, F. (2003) Mathematical Methods in Physics and Engineering with Mathematica. CRC Press, Boca Raton. http://dx.doi.org/10.1201/9780203502600

[14] Khalil, S.M. (1996) Eigenvalues of Relaxed Toroidal Plasmas of Arbitrary Sharp Edged Cross Section. 6th Conference of Nuclear Sciences and Applications, Cairo, 15-20 March 1996, 252.

[15] Cap, F. (1996) Collocation Methods in Plasma Physics. 4th Workshop on Plasma and Laser Physics, Cairo, 26-29 February 1996.

[16] Khalil, S.M. and Altuijri, R.A. (2009) Relaxed Plasma State in Compact Tori. Journal of Fusion Energy, 28, $275-281$. http://dx.doi.org/10.1007/s10894-008-9187-5

[17] Khalil, S.M., Alomayrah, N.A. and Altuijri, R.A. (2012) Zero Flux Eigenvalues of Relaxed Axisymmetric Compact Tokamaks (CT’s). Journal of Fusion Energy, 31, 1-6. http://dx.doi.org/10.1007/s10894-011-9434-z

[18] Hole, M.J., Hudson, S.R. and Dewar, R.L. (2006) Stepped Pressure Profile Equilibria in Cylindrical Plasmas via Partial Taylor Relaxation. Journal of Plasma Physics, 72, 1167-1171. http://dx.doi.org/10.1017/S0022377806005861

[19] Hudson, S.R., Hole, M.J. and Dewar, R.L. (2007) Eigenvalue Problems for Beltrami Fields Arising in a Three-Dimensional Toroidal Magnetohydrodynamic Equilibrium Problem. Physics of Plasmas, 14, Article ID: 052505. http://dx.doi.org/10.1063/1.2722721

[20] Gray, T., Brown, M.R. and Dandurand, D. (2013) Observation of a Relaxed Plasma State in a Quasi-Infinite Cylinder. Physical Review Letters, 110, Article ID: 085002. http://dx.doi.org/10.1103/PhysRevLett.110.085002

[21] Browning, P.K., Stanier, A., Ashworth, G., McClements, K.G. and Lukin, V.S. (2014) Self-Organization during Spherical Torus Formation by Flux Rope Merging in the Mega Ampere Spherical Tokamak. Plasma Physics and Controlled Fusion, 56, Article ID: 064009. http://dx.doi.org/10.1088/0741-3335/56/6/064009

[22] Lloyd, B., Ahn, J.-W., Akers, R.J., Appel, L.C., Applegate, D., Axon, K.B., et al. (2014) MAST and the Impact of Low Aspect Ratio on Tokamak Physics. Plasma Physics and Controlled Fusion, 46, B477-B494. http://dx.doi.org/10.1088/0741-3335/46/12B/040 